When does a populated place become overpopulated? By what criteria is it judged to be so? These are questions that are rarely asked or answered in precise terms. A clear discussion of them from the medical profession is overdue.

1 Fifth Report from the Select Committee on Science and Technology

2 Registrar General's Statistical Review of England and Wales for the year 1970, Part 2, Tables, Population. London, H.M.S.O.,

3 British Medical fournal Supplement, 1972, 3, 103.

\section{Medical Radiations}

Next to the natural background, which we cannot alter, medical radiology is the largest source of radiation to the population in developed countries. The incidental dangers to staff and patients are generally difficult to measure-or even to recognize-but their control is important, if only because doctors should keep their own houses in order before pronouncing, as they are often expected to do, on fall-out, nuclear power, and other hazards of modern life.

The Code of Practice, issued by the Health Departments for the guidance of hospital authorities and individual radiation users, now appears in a new edition. ${ }^{1}$ As in earlier formulations, the guiding principle is that radiation doses to staff and patients should be no greater than is necessary to produce a satisfactory result. No significant changes are proposed in the maximum permissible dose levels (which few radation workers ever approach) or in the other protective measures defined at the last revision in 1964, but additional administrative measures are recommended. Patients discharged from hospital while carrying substantial amounts of radioactivity will in future be supplied with instruction cards dealing with radiation and contamination hazards. Advice is given on the procedure (already used by some employing authorities) for deciding whether particular classes of radiation workers should carry film badges or whether their safety is adequately maintained by regular environmental monitoring. More detailed arrangements for record keeping and for annual reviews of safety are also prescribed.

The British way of dealing with radiation hazards is a characteristic compromise between individual freedom and legislative restriction, but it is undoubtedly effective, whether judged by the reduction of doses to staff and patients or by the satisfied state of public opinion. This success story might usefully be studied in relation to the contentious problems created by more recently recognized forms of environmental pollution.

\section{Code of Practice for the Protection of Persons against Ionizing Radiations arising from Medical and Dental Use, 3rd edn. London, H.M.S.O., 1972 .}

\section{Spongy Change in the Brain}

"Spongy change" is the description given to the distinctive appearance of the brain in a variety of neurological diseases. The terms "spongiform encephalopathy" and "status spongiosus" are also used. The appearance may be suffciently gross to be visible to the naked eye, but the terms are applied as well to the microscopic appearance of the scattered spaces which are presumed to result from the loss of brain substance. Sometimes only one cell type or cell component is picked out. F. Seitelberger's definition of status spongiosus as "an alteration of the central nervous tissue in the form of a spongy loosening presenting a threedimensional network of fibrillar tissue components and appearing as a system of vesicular cavities filled with fluid"1 is somewhat stylized. It is also an extreme definition of a process which may be both fragmentary and focal.

Spongy changes can be observed in a variety of aetiologically different diseases such as Alzheimer's disease, neuro-axonal dystrophy, certain neuronal lipidoses, Hallervorden-Spatz disease, arteriosclerosis, hypertension, and some post-infectious, post-inflammatory syndromes. ${ }^{1}$ The exact relationship with derangements of intracellular structure and metabolism as seen primarily in cerebral oedema has been widely debated. ${ }^{2}$ Concurrently with the spongy change, however, other cellular reactions also occur-for instance, glial proliferation, sometimes with formation of fibrillary astrocytes, loss of myelin, phagocytosis, and loss of cells Though these striking changes have prompted speculation about their causes, the study of them so far has done no more than point to a classification of degenerative diseases.

A recent paper ${ }^{3}$ reporting so-called "spongiform encephalopathy" in six patients dying with Jakob-Creutzfeldt disease is a reminder of how, in this disease, a transmissible agent has been detected. ${ }^{4}$ The classical histological features of viral encephalitis with inclusion bodies are lacking, and at present the precise pathogenesis of this and other supposed slow infective encephalopathies is unknown. It is not known whether the agent is a virus in the accepted sense, and no surprise, therefore, that treatment with idoxyuridine (effective only against DNA viruses) has been unsuccessful.

By contrast there is a rare progressive degenerative disease in infants, known as spongy degeneration of the brain, or Canavan's disease, 56 whicn is genetically determined as an autosomal recessive condition, mainly in patients of Jewish extraction. An inborn metabolic error is almost certainly the cause of this disease, which presents at about the sixth month, with arrest of mental development and progressive loss of skills. Against a background of hypotonia extensor spasms occur, and though progressive enlargement of the head may be the result of obstructive hydrocephalus in some patients it is more commonly due to true megalencephalus of a degree sometimes sufficient to cause separation of sutures. Optic atrophy occurs early, but, though the rest of the clinical picture closely resembles the early stages of classical Tay-Sachs disease, neither the characteristic enzymic abnormalities nor a cherry red spot at the macula have ever been reported at any stage of the disease. Gradually the clinical picture is dominated by decerebration with some dystonic features, and most patients die during the second year of the affliction. There are no pathognomonically helpful investigations in life-in particular, spinal fluid is usually normal. At necropsy the brain has the consistency as well as the appearance of a sponge, particularly the white matter. These changes may occur throughout the neuraxis, but nerve roots are spared. Tiny cavities are concentrated in deeper structures round small blood vessels, and vacuoles develop in cytoplasm of both neurones and glia.

These particular changes have been considered to be specific, but essentially similar changes may occur in hyperglycinaemia, ${ }^{7}$ arginosuccinic acidaemia, ${ }^{8}$ homocystinuria, ${ }^{9}$ 